\title{
Dynamique de colonisation des voies navigables du bassin de la Seine par Chelicorophium curvispinum (Crustacea : Amphipoda)
}

\section{Dynamics of colonization by Chelicorophium curvispinum (Crustacea: Amphipoda) in navigable waterways of the Seine basin}

\author{
J. Souben ${ }^{(1)}$, J. Loiseau ${ }^{(1)}$, M.-C. Roger ${ }^{(2)}$ \\ (1) Hydrosphère, 2 avenue de la mare (Zl des béthunes), 95310 St Ouen l'Aumone, France \\ jeremie.souben@gmail.com,www.hydrosphère.fr \\ (2) IRSTEA UR Milieux Aquatiques, Écologie et Pollutions - Laboratoire Dynamiques, indicateurs et modèles \\ en écohydrologie, 3 bis quai Chauveau, 69336 Lyon Cedex, France
}

Résumé - La synthèse bibliographique de données hydrobiologiques entre 1997 et 2008 (141 stations) et la campagne de terrain menée en 2009 (29 stations) ont permis de réaliser un premier état des lieux de la présence de Chelicorophium curvispinum dans les voies navigables du bassin de la Seine, depuis ses premières observations en 1998 dans les canaux de Picardie. En 2009, C. curvispinum est présent dans les bassins de l'Oise et de l'Aisne aval, ainsi qu'aux abords de l'agglomération parisienne. II demeure absent, ou très rare et isolé, sur le reste du bassin. L'analyse des différentes données montre que $C$. curvispinum a d'abord colonisé le bassin de l'Oise (et l'Aisne) par une lente dérive via les canaux du Nord. La population présente à proximité de l'agglomération parisienne est apparue plus tardivement de manière isolée et pourrait être liée au trafic fluvial (eaux de ballast). Cette situation (répartition disparate, densités modérées, colonisation lente, pas d'effet majeur sur la macrofaune autochtone) est assez atypique en comparaison des données observées sur d'autres bassins hydrographiques (Rhin, Meuse, Moselle...) ou pour d'autres néozoaires ponto-caspiens présents dans le bassin de la Seine comme Dikerogammarus villosus notamment. À ce titre, le caractère invasif de $C$. curvispinum dans le bassin de la Seine peut être discuté.

Mots-clés - Chelicorophium curvispinum, crustacé, néozoaire, espèce invasive, bassin Seine Normandie

Abstract - The literature review of hydrobiological data between 1997 and 2008 (141stations) and the field surveys conducted in 2009 (in 29 stations) allowed to achieve an initial inventory of Chelicorophium curvispinum occurrences in navigable waterways of the Seine basin since its first observations in 1998 in the Picardy canals. In 2009, C. curvispinum was present in the downstream part of Oise and Aisne sub-basin and nearby Paris. It remains 


\begin{abstract}
absent, or present in very rare and isolated cases in the rest of the Seine basin. Analysis of data shows that $C$. curvispinum first colonized the Oise sub-basin (and Aisne basin) with a slow drift via the North canals. The population present nearby the city of Paris appeared later in an isolated way and could be linked to the river traffic (through ballast waters). This situation (patchy distribution, moderate densities, slow colonization abilities, no major effects on indigenous macrofauna) is rather unusual compared to data observed in others basins (Rhine, Meuse, Moselle...) or to other Ponto-Caspian neozoaires present in the Seine basin (such as Dikerogammarus villosus). In this regard, the invasive feature of $C$. curvispinum in the Seine basin could be questioned.
\end{abstract}

Key words - Chelicorophium curvispinum, crustacean, neozoa, invasive species, Seine basin

\section{INTRODUCTION}

Originaire des milieux saumâtres du bassin Ponto-Caspien, C. curvispinum, crustacé amphipode de la famille des Corophiidae (Fig. 1), a étendu son aire de répartition vers l'Europe de l'ouest au début du $X X^{\mathrm{e}}$ siècle. II a d'abord colonisé les rivières de Pologne dans les années 1920 (Jazdzewski \& Konopacka, 2000) puis les fleuves d'Europe centrale comme l'Oder, la Vistule et l'Elbe (Jazdzewski,1980). II apparaît en Allemagne de l'ouest dans les années 1950 et arrive dans le canal de Dortmund en 1977 (Herhaus, 1978). II envahit ensuite le Rhin entre 1982 et 1989 (Van den Brink et al., 1989), poursuit son extension vers le nordouest en colonisant la Meuse (Wouters, 1985 ; Khalanski, 1997) et la Moselle (Schöll, 1990 ; Bachmann et al., 1997 ; Kallenbach, 2000) puis vers le sud où il est observé dans la Saône en 1991 (Genin, 1992) et dans le Rhône au début des années 2000 (Dessaix \& Fruget, 2008).
Cette extension s'est faite par le biais des grands fleuves et des canaux de navigation (Jazdzweski, 1980 ; Bachmann et al., 2001). Ainsi, le canal du Danube au Rhin deviendra la route privilégiée de migration des néozoaires ponto-caspiens dès sa mise en service en 1992 (Bij de Vaate et al., 2002). La dérive, mais aussi le transport par les eaux de ballast, ont joué un rôle majeur dans la dispersion du C. curvispinum (Pygott \& Douglas, 1989) et expliquent notamment sa présence en Angleterre dès les années 1930 (Moon, 1970).

Chelicorophium curvispinum est considéré comme une espèce invasive dans la plupart des cours d'eau où il s'est implanté. Son potentiel invasif dans les grands cours d'eau est favorisé par une fécondité élevée ( 3 génération par an) ainsi qu'une tolérance au batillage (Pygott \& Douglas, 1989) et aux variations de salinité (Balyss \& Harris, 1988).

Le bassin de la Seine, situé à la périphérie de zones déjà colonisées et traversé par de nombreux canaux où le 


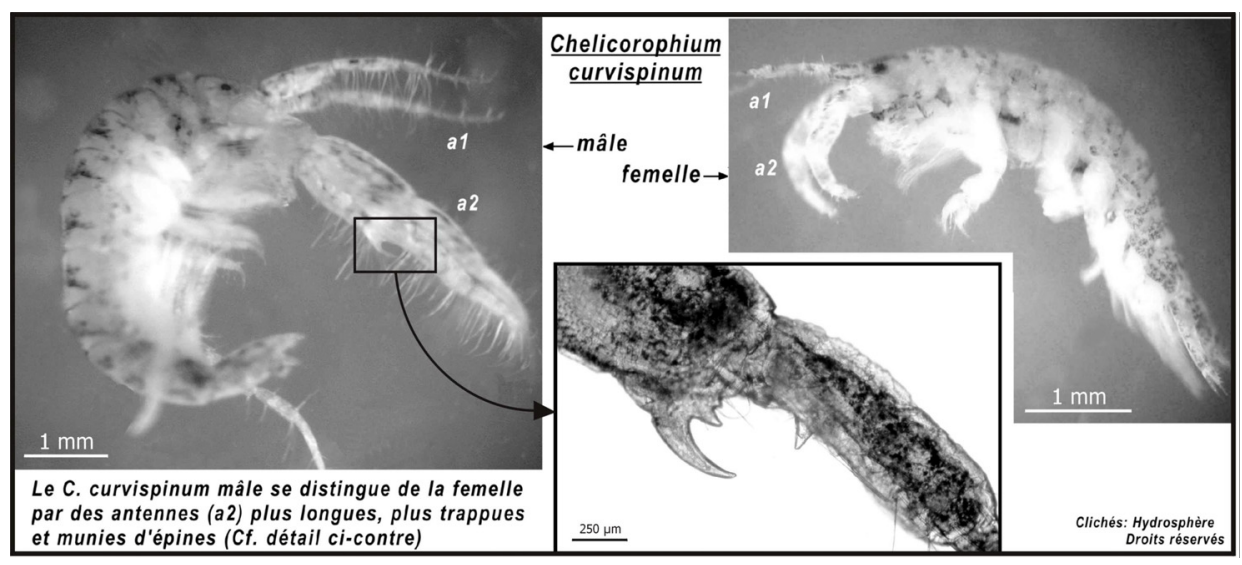

Fig. 1. Photographies de Chelicorophium curvispinum ainsi que l'un de ses principaux critères de reconnaissance (épine sur la paire d'antenne A2).

Fig. 1. Pictures of Chelicorophium curvispinum as well as one of its main criteria of recognition (thorn on the pair of antenna A2).

trafic fluvial est important (liaisons entre Paris et l'Atlantique (via la Seine) et les grands ports de la Manche (via l'Oise et les canaux du Nord), apparaît particulièrement exposé à une colonisation rapide par ce crustacé. Ce travail a pour objectif de procéder, à partir de données hydrobiologiques, à un historique de la répartition de cette espèce dans les voies navigables du bassin de la Seine.

\section{MATÉRIEL ET MÉTHODES}

La recherche du C. curvispinum dans le bassin de la Seine s'est focalisée sur les grands cours d'eau navigués (Seine, Marne, Oise et Aisne) et les principaux canaux qui le traversent.

Une synthèse bibliographique a permis de dresser un premier état des lieux à partir des résultats hydrobiologiques (IBGA) de l'Agence de l'eau Seine Normandie, du GIP Seine-aval et de la société Hydrosphère, soit un total de 141 stations échantillonnées à des fréquences variables, entre 1997 et 2008 (Fig. 2). Pour chaque IBGA, 8 prélèvements rivulaires, un prélèvement par drague et 2 substrats artificiels constituaient l'unité d'effort pour l'évaluation de l'indice.

En 2009, des prospections ciblées sur le $C$. curvispinum ont été réalisées sur 29 stations pour compléter les données bibliographiques (suivi de populations existantes, recherche de nouvelles voies de colonisation, ...). Ces 29 stations ont été échantillonnées à l'aide de substrats artificiels tels que ceux utilisés lors de la réalisation d'IBGA (bourriche de grillage de $20 \mathrm{~cm} \times 30 \mathrm{~cm}$ remplie de 3 pierres plates et de $25 \mathrm{~m}$ de corde sisal). L'analyse détaillée des données bibliographiques a montré que ces dispositifs étaient efficaces pour l'échantillonnage de $C$. curvispinum (Souben, résultats non publiés) et permettaient une bonne 


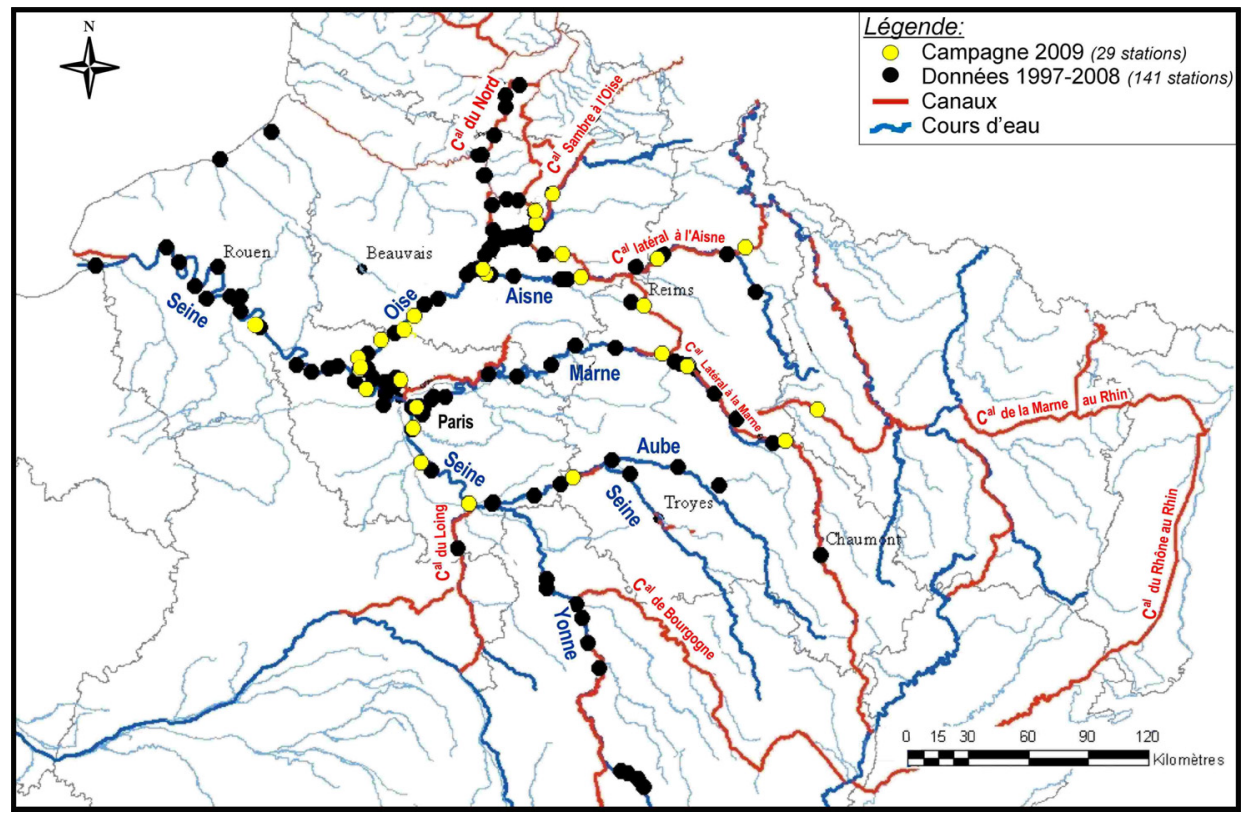

Fig. 2. Localisation des données utilisées pour le recensement du C. curvispinum dans le bassin de la Seine depuis 1997.

Fig. 2. Localization of the data used for the inventory of $C$. curvispinum in the Seine basin since 1997.

reproductibilité des efforts de captures entre les différentes stations.

En l'occurrence, deux substrats ont été disposés sur chaque station pendant trois semaines (de la fin avril 2009 à la mi-mai 2009). Les organismes récoltés ont été fixés au formol in situ puis triés, déterminés et comptés en laboratoire. Les résultats présentés ici ne concernent que $C$. curvispinum.

\section{RÉSULTATS}

Le bassin Seine Normandie a été découpé en 5 sous bassins: l'Oise, l'Aisne, la Marne, la Seine en amont de Paris et la Seine de Paris à son embouchure. Les résultats ci-dessous combinent :
- les données bibliographiques (1997-2008) où l'effort de capture est exprimé en nombre d'individus/ IBGA correspondant au nombre d'individus récoltés à l'aide de 8 prélèvements rivulaires, un prélèvement par drague et 2 substrats artificiels,

- les résultats obtenus en 2009 (Tab. I) où l'effort de capture est exprimé en nombre d'individus capturés dans les deux substrats artificiels.

\subsection{Sous-bassin de l'Oise}

Il comprend l'Oise sur l'ensemble de son cours et les canaux associés (canal du Nord, canal de Saint-Quentin, canal 
Tableau I. Liste des sites échantillonnés en 2009 et nombre de $C$. curvispinum récoltés sur les 2 substrats artificiels.

Table I: List of the stations sampled in 2009 and number of $C$. curvispinum collected on 2 artificial substrata.

\begin{tabular}{|c|c|c|c|c|}
\hline Cours d'eau & Ville & Navigable & $\begin{array}{c}\text { Temps de } \\
\text { colonisation } \\
\text { (jours) }\end{array}$ & $\begin{array}{c}\text { Nombre de } C \text {. } \\
\text { curvispinum }\end{array}$ \\
\hline \multicolumn{5}{|c|}{ Bassin de l'Oise } \\
\hline Canal Sambre à Oise & Berthenicourt & Oui & 20 & 18 \\
\hline Canal St-Quentin & Mennessis & Oui & 20 & 12 \\
\hline Canal latéral à l'Oise & Condren & Oui & 20 & 1 \\
\hline Oise & Saint-Leu d'Esserent & Oui & 20 & 23 \\
\hline Oise & Boran sur Oise & Oui & 20 & 7 \\
\hline Oise & Isle Adam & Oui & 20 & 7 \\
\hline Oise & Cergy & Oui & 24 & 0 \\
\hline Oise & $\begin{array}{l}\text { Conflans Saint } \\
\text { Honorine }\end{array}$ & Oui & 24 & 2 \\
\hline \multicolumn{5}{|c|}{ Bassin de l'Aisne } \\
\hline Canal des Ardennes & Montgon & Oui & 20 & 0 \\
\hline Canal latéral à l'Aisne & Asfeld & Oui & 20 & 0 \\
\hline Canal Oise-Aisne & Pinon & Oui & 20 & 70 \\
\hline Canal latéral à l'Aisne & Cys la Commune & Oui & 20 & 9 \\
\hline Aisne & Rethondes & Oui & 20 & 46 \\
\hline Aisne & Francport & Oui & 20 & 0 \\
\hline \multicolumn{5}{|c|}{ Bassin de la Marne } \\
\hline Canal Marne au Rhin & Bar le duc & Oui & 20 & 0 \\
\hline Canal Marne à Saône & Saint-Dizier & Oui & 20 & 0 \\
\hline $\begin{array}{c}\text { Canal latéral à la } \\
\text { Marne }\end{array}$ & Recy & Oui & 20 & 0 \\
\hline Marne & Saint-Gibrien & Non & 20 & 0 \\
\hline Canal Marne à Aisne & Reims & Oui & 20 & 0 \\
\hline $\begin{array}{c}\text { Canal latéral à la } \\
\text { Marne }\end{array}$ & Tours sur Marne & Oui & 20 & 4 \\
\hline Marne & Maisons Alfort & Oui & 23 & 12 \\
\hline \multicolumn{5}{|c|}{ Bassin de la Seine amont } \\
\hline Seine & Nogent sur Seine & Oui & 20 & 0 \\
\hline Loing & Saint-Mammès & Oui & 20 & 72 \\
\hline Seine & Morsang sur Seine & Oui & 20 & 0 \\
\hline Seine & Ablon sur Seine & Oui & 23 & 87 \\
\hline Seine & Nogent sur Seine & Oui & 20 & 0 \\
\hline \multicolumn{5}{|c|}{ Bassin de la Seine aval } \\
\hline Seine & Saint-Denis & Oui & 24 & 0 \\
\hline Seine & Le Pecq & Oui & 21 & 0 \\
\hline Seine & Andrésy & Oui & 24 & 0 \\
\hline Seine & Courcelles & Oui & 24 & 0 \\
\hline
\end{tabular}




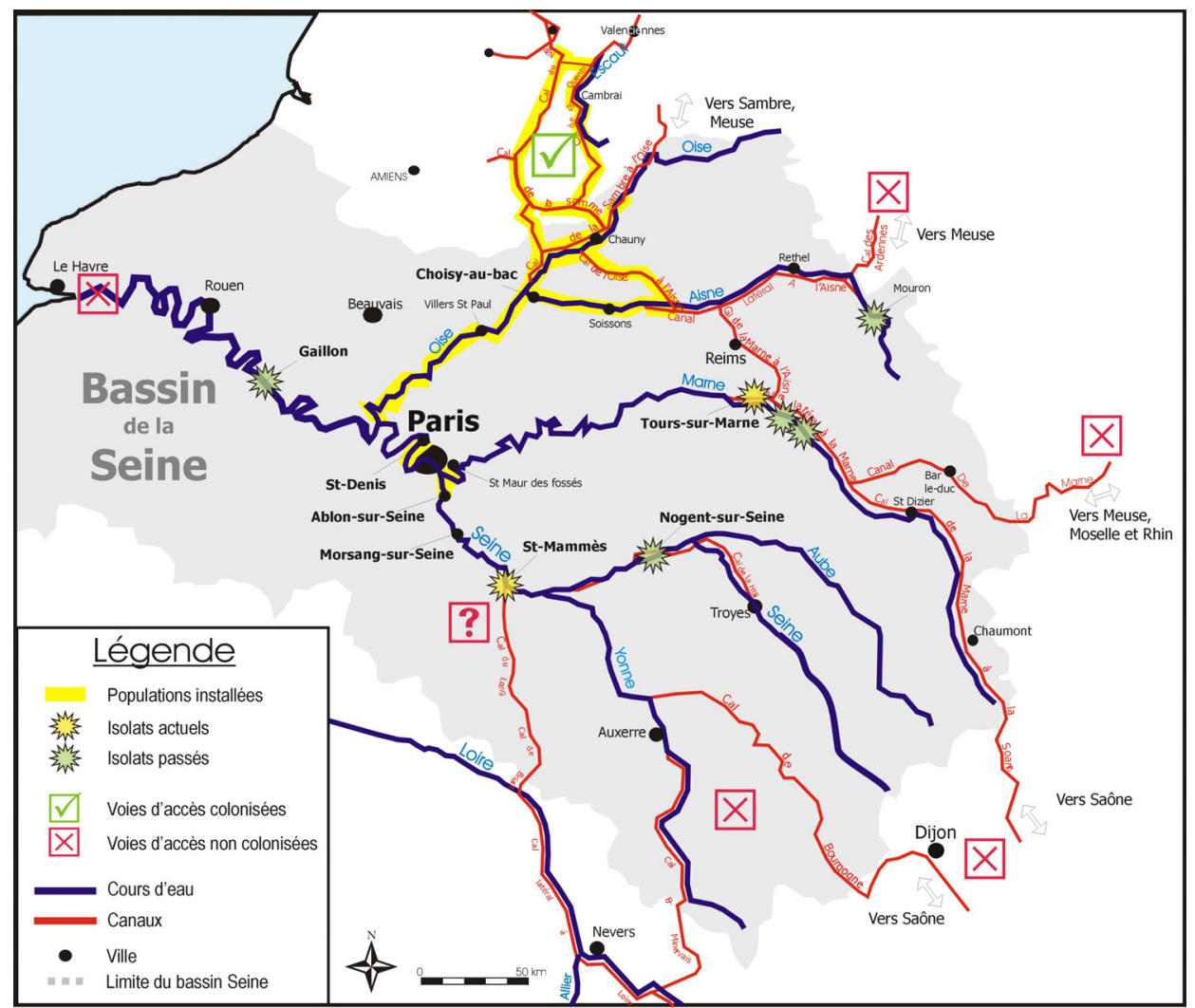

Fig. 3. État des populations de C. curvispinum en 2009 dans le bassin de la Seine.

Fig. 3. Inventory of the populations of $C$. curvispinum in 2009 in the Seine basin.

de la Sambre à l'Oise, canal latéral à l'Oise et canal de l'Oise à l'Aisne).

Le C. curvispinum a été observé pour la première fois en 1998 dans le canal latéral à l'Oise et le canal de l'Oise à l'Aisne. Toutefois, les abondances observées (jusqu'à 5000 ind./IBGA) plaident pour une colonisation bien antérieure.

La synthèse des données entre 1997 et 2008 indique que le C. curvispinum est présent depuis l'an 2000 dans les canaux et l'Oise naviguée entre Chauny et Villers-St-Paul (sur l'Oise médiane) (Fig. 3), avec des abondances de l'ordre du millier d'individus/IBGA suivant les années. En revanche, il n'apparaît dans l'Oise aval qu'en 2005, à proximité de sa confluence avec la Seine. Ces résultats confirment la colonisation du cours de l'Oise par les voies navigables en amont du bassin (canaux puis Oise canalisée). Le canal du Nord est, jusqu'en 2008, la seule voie d'entrée fonctionnelle du C. curvispinum dans ce bassin (Fig. 3).

Les résultats de la campagne 2009 ont révélé la présence du $C$. curvispinum dans les canaux de la Sambre à 
l'Oise et de Saint-Quentin, dans lesquels il n'avait pas été trouvé en 2000. Concernant l'Oise aval, les cinq stations échantillonnées entre SaintLeu d'Esserent et la confluence avec la Seine témoignent d'une population instable avec des effectifs faibles et fluctuants suivant les stations (2 à 23 individus pour les deux substrats artificiels).

\subsection{Sous-bassin de l'Aisne}

II comprend les grands cours d'eau de l'Aisne et de la Vesles ainsi que les canaux associés (canal de l'Oise à l'Aisne, canal latéral à l'Aisne, canal des Ardennes et canal de la Marne à l'Aisne).

Le $C$. curvispinum est observé pour la première fois sur le canal de l'Oise à l'Aisne en 1998 (cf. Sect. 3.1). Sa présence y est confirmée deux ans plus tard.

Dans l'Aisne, la seule population installée se limite, jusqu'en 2007, aux abords de sa confluence avec l'Oise (Choisy-au bac). L'année 2007 marque un tournant: le $C$. curvispinum est recensé avec des effectifs importants (jusqu'à 3000 ind./IBGA) sur quatre stations de l'Aisne aval (entre le canal de l'Oise à l'Aisne et la confluence avec l'Oise - Fig. 3). Cette expansion est confirmée en 2008.

En revanche, C. curvispinum n'a jamais été recensé dans l'Aisne en amont du canal de l'Oise à l'Aisne (Fig. 3). Il en est de même dans le canal latéral à l'Aisne et le canal de la Marne à l'Aisne.

Les inventaires menés en 2009 visaient à suivre l'état des populations du secteur aval et vérifier l'absence du C. curvispinum sur le secteur amont.
Les résultats obtenus en 2009 confirment les données bibliographiques:

- C. curvispinum est bien présent dans le bassin aval de l'Aisne, en aval de la confluence avec le canal de l'Oise à l'Aisne (46 individus pour les deux substrats artificiels à Rethondes), y compris dans le canal latéral à l'Aisne où il a été échantillonné pour la première fois en 2009 (9 individus pour les deux substrats artificiels à Cys la Commune).

- Il est absent à l'amont (Aisne et canal latéral), y compris dans les voies d'accès potentielles : canal de la Marne à l'Aisne et Canal des Ardennes (en communication avec la Meuse).

\subsection{Le sous-bassin de la Marne}

Ce sous bassin comprend la Marne, le canal latéral à la Marne, le canal de la Marne au Rhin et le canal de la Marne à la Saône.

C. curvispinum est recensé pour la première fois dans la Marne en 1999, aux environs de Châlons-en-Champagne (Fig. 3), mais il y est peu abondant (1 ind./IBGA sur chacune des deux stations échantillonnées). Les effectifs observés sur ces stations seront plus conséquents en 2003, (30 à 200 ind./IBGA) mais déclineront en 2004 (1 ind./IBGA) et ne seront pas retrouvés dans les inventaires suivants (2005, 2006 et 2009).

Entre 1997 et 2009, C.curvispinum n'est présent que dans les 10 derniers kilomètres du cours de la Marne entre Saint-Maur-des-Fossés et la confluence avec la Seine où il apparaît en 2004 (hors population recensée et disparue 
autour de Châlons-en-Champagne). II y sera retrouvé en 2007, 2008 et 2009 avec des effectifs toujours réduits ( $<15$ individus).

Concernant les canaux, les deux informations importantes sont les suivantes :

- C. curvispinum est absent des canaux reliant la Marne aux bassins de la Meuse, Moselle et Saône, pourtant colonisés depuis au moins 1995 (Khalanski, 1997). Cette absence, constatée en 2000, est confirmée en 2009 (aucun individu à Bar le duc et à Saint-Dizier).

- C. curvispinum a été recensé en 2000 et 2009 dans le canal latéral à la Marne à Tours/Marne (à proximité de la confluence entre le canal et la Marne). Cette population est réduite $(<5$ individus pour les 2 substrats artificiels) et isolée (absence en amont et en aval de Tours/Marne) et peut être qualifiée d'isolat (cf. cidessus).

\subsection{Sous-bassin Seine amont}

Le sous-bassin Seine amont comprend la Seine de Troyes à Paris, l'Aube, l'Yonne, le canal de Bourgogne et le canal du Loing.

Les données bibliographiques disponibles sur ce sous bassin entre 1997 et 2002 se limitent aux résultats hydrobiologiques (IBGA) obtenus en 1999 en aval immédiat du barrage d'Ablon sur Seine, à $15 \mathrm{~km}$ en amont de l'agglomération Parisienne. C. curvispinum est absent dans ce secteur cette année là mais il y apparaît pour la première fois en 2003. Les inventaires ultérieurs (2004 à 2007) indiquent des densités allant de 70 à 600 ind./IBGA/ an. II s'agit, jusqu'en 2009, de la seule population pérenne de $C$. curvispinum du bassin de la Seine en amont de Paris.

Aux environs de Nogent sur Seine, un seul individu a été récolté en 2007 et 2008 (Fig. 3) mais aucun en 2009. Cette population semble donc correspondre à un isolat passé.

La campagne 2009 a mis en évidence une nouvelle population de $C$. curvispinum dans le canal du Loing à Saint-Mammès, à proximité de sa confluence avec la Seine (Fig. 3). Les 72 individus récoltés sur les deux substrats témoignent d'une population significative.

Enfin, il est intéressant de noter qu'aucun $C$. curvispinum n'a jamais été signalé dans l'Yonne et le canal de Bourgogne, pourtant en liaison avec la Saône où il est présent depuis 1991.

\subsection{Sous-bassin Seine aval}

Ce sous-bassin concerne uniquement le cours de la Seine de Paris jusqu'à son embouchure. C. curvispinum n'a jamais été observé entre 1997 et 2004 malgré 25 campagnes IBGA réparties sur l'ensemble du cours aval. La première observation date de 2005 , avec 3 ind./IBGA récoltés à SaintDenis, à $10 \mathrm{~km}$ en aval de Paris. II n'avait pas été récolté sur cette station en 2001 ni 2004 et les campagnes ultérieures témoignent d'une population instable (absent en 2006 et 2007, 6 ind./IBGA en 2008, absent en 2009).

En revanche, trois stations échantillonnées entre Paris et Saint-Denis en 2007 et 2008 confirment la présence 
de $C$. curvispinum en aval immédiat de l'agglomération parisienne, notamment en 2008 (130 à 260 ind ./IBGA).

C. curvispinum est toujours absent, en 2009, du reste du cours de la Seine. À noter toutefois la présence sur le secteur de Gaillon (Fig. 3) de 5 individus récoltés sur deux stations en 2007 mais aucun en 2008 et 2009.

\subsection{Bilan à l'échelle du bassin Seine}

La synthèse des données recueillies sur l'ensemble du bassin de la Seine entre 1997 et 2009 montre que C. curvispinum est aujourd'hui bien installé dans l'Oise, l'Aisne aval et la Seine aux environs de l'agglomération parisienne (Fig. 3).

En revanche, il n'est présent que de façon ponctuelle (isolat) dans le reste du bassin puisque seules deux populations représentées par uniquement quelques individus ont été observées dans le canal latéral à la Marne (Tours/ Marne) et le canal du Loing (SaintMammès). C. curvispinum n'a été observé que de façon temporaire dans la Marne à Châlons-en-Champagne (entre 1999 et 2004), la Seine à Nogent (2007 et 2008) et à Gaillon (2007).

\section{DISCUSSION}

Les résultats présentés ont permis de retracer l'histoire de l'introduction et de la dispersion de C. curvispinum dans le bassin de la Seine entre 1997 et 2009.

Ce crustacé est arrivé dans le bassin de la Seine au plus tard en 1998 par les canaux du Nord et de la Somme. II a progressivement colonisé le bassin de l'Oise d'amont en aval, jusqu'à atteindre la confluence de la Seine en 2005. Dans le même temps, il s'est propagé de l'Oise vers le bassin de l'Aisne via le canal de l'Oise à l'Aisne (dans lequel il est présent dès 1998). II a ensuite dévalé l'Aisne jusqu'à la confluence avec l'Oise (Fig. 3). La colonisation de cette partie de l'Aisne est pratiquement achevée en 2007.

L'expansion du C. curvispinum dans le bassin de l'Oise s'est donc essentiellement faite de l'amont vers l'aval. C. curvispinum n'a manifestement pas réussi à coloniser l'amont de l'Aisne comme il l'a fait dans la Meuse et la Moselle. Les remontées observées se limitent à quelques kilomètres aux abords des confluences (Aisne/ Oise, canal latéral à l'Aisne/Aisne).

Enfin, le $C$. curvispinum a pratiquement stoppé sa migration vers l'aval depuis 2005, que ce soit vers la Seine (à partir de l'Oise) ou la Marne (à partir de l'Aisne).

Ainsi, la population de C. curvispinum identifiée à proximité de l'agglomération parisienne depuis 2003 (en amont) et 2005 (en aval) a certainement été introduite à partir de l'activité portuaire autour de la capitale (transfert par les eaux de ballast). Elle se trouve au carrefour des principales voies navigables du bassin de la Seine, qui sont autant de voies d'accès potentielles pour $C$. curvispinum (Fig. 3 ).

Bien que $C$. curvispinum n'ait pas colonisé la Marne, la Seine amont et aval, les isolats (passés et présents) témoignent de son transfert possible par ces voies navigables en relation avec des bassins où il est historiquement abondant (Meuse, Moselle, Saône). 
Enfin, les données les plus récentes montrent que cette population ne parvient pas à remonter plus en amont dans la Seine ou la Marne et n'est pas présente dans la Seine en aval de Saint-Denis (Fig. 3).

Cette expansion lente et limitée de C. curvispinum dans le bassin de la Seine est relativement inattendue et contraste singulièrement avec les résultats observés dans la Meuse, la Moselle et le Rhin. C. curvispinum n'a mis que sept années pour envahir le Rhin (Van den Brink et al., 1989) et seulement deux pour devenir l'espèce majoritaire de son cours aval (Den Hartog et al., 1992 \& Paffen et al., 1994), avec des densités pouvant atteindre 642000 individus par $\mathrm{m}^{2}$ (Rajagopal et al., 1998). En comparaison, il lui aura fallu également sept années pour coloniser le cours médian et aval de l'Oise (soit un linéaire 3 à 4 fois inférieur au Rhin). Les densités maximales évaluées dans l'Oise sont de l'ordre de 80000 à 160000 individus $/ \mathrm{m}^{2}$ (principalement dans les franges racinaires) et $C$. curvispinum ne représente alors que $25 \%$ à $50 \%$ des invertébrés récoltés (protocole IBGA).

Plusieurs pistes peuvent être proposées pour expliquer cette colonisation relativement modérée des grands cours d'eau du bassin de la Seine par le C. curvispinum:

Les effets de la pollution des eaux peuvent être envisagés mais la sensibilité du $C$. curvispinum demeure mal connue. Elle est évoquée pour expliquer sa disparition dans les bassins de la Volga (Jazddzweski, 1980) mais le C. curvispinum est également considéré comme très résistant à la pollution et capable de supporter des effluents industriels et miniers. En la matière, les résultats hydrobiologiques obtenus de part et d'autre de rejets industriels du bassin de la Seine mettent, dans certains cas, en évidence une sensibilité du C. curvispinum à la pollution, que ce soit à l'échelle locale (rejet) ou de façon plus globale (cours d'eau, canaux).

La compétition interspécifique pourrait également affecter la cinétique de dispersion du C. curvispinum. Au contraire des canaux de navigation où l'artificialisation du lit entraîne une simplification importante des biocénoses à la faveur des espèces les plus opportunistes et résistantes telles que certains néozoaires, la relative naturalité des grands cours d'eau du bassin Seine favorise la compétition interspécifique entre les peuplements macrobenthiques (crustacés notamment). La première intrusion du $C$. curvispinum par le canal du Nord va dans ce sens, de même que son absence loin des grandes agglomérations (secteurs naturels). En revanche, son absence dans les canaux de l'est du bassin demeure difficile à expliquer.

Enfin, il faut citer le cas du Dikerogammarus villosus, crustacé invasif également originaire de la région pontocaspienne. Ce gammaridae carnivore, dénommé "crevette tueuse » par les anglo-saxons, est réputé pour exercer une prédation importante sur les crustacés autochtones. Ainsi, le suivi des populations de micro-crustacés de la Moselle montre une chute brutale des effectifs de $C$. curvispinum l'année d'apparition du $D$. villosus (Kallenbach et al., 2000). Or, le D. villosus a été observé pour la première fois en lle-de-France en 2000 et a supplanté en quelques années le gammare 
autochtone (Gammarus pulex) jusqu'à l'éradiquer des grands cours d'eau de la région.

Contrairement aux bassins de la Meuse et Moselle, le $D$. villosus est arrivé dans la région lle-de-France avant le $C$. curvispinum, créant dans ces cours d'eau des conditions sûrement peu propices à l'expansion de ce dernier.

\section{Perspectives}

La répartition singulière de $C$. curvispinum dans le bassin de la Seine soulève plusieurs questions :

- La colonisation des voies navigables du bassin par $C$. curvispinum estelle en expansion ou en déclin?

Les résultats de cette étude suggèrent que $C$. curvispinum est en cours d'expansion mais son évolution à moyen et long terme demeure imprévisible, tant du point de vue spatial que temporel. Les densités récoltées dans le cadre de l'étude sont globalement stables pour les populations historiquement installées (Oise, Aisne aval) mais trop incomplètes sur les autres stations pour ébaucher une tendance. Au niveau spatial, il serait nécessaire d'affiner le maillage des stations aux limites des populations actuelles (notamment en région parisienne) afin de suivre son évolution.

- Pourquoi C. curvispinum demeure absent des voies navigables en communication avec les bassins de la Meuse et de la Moselle?

Ce résultat très inattendu demeure difficilement explicable, vu l'importance des populations de $C$. curvispinum recensées dans ces grands cours d'eau de l'est de la France depuis les années 1995-2000. II serait intéressant d'évaluer les effectifs de $C$. curvispinum aux abords des jonctions entre ces fleuves (Meuse/Moselle) et les canaux (canal de la Marne au Rhin notamment) pour mieux comprendre l'absence de dissémination du crustacé par ces voies navigables. L'analyse des flux de navigation (type de bateaux, fréquences de transport...) pourrait également apporter des éléments de réponses.

- La population observée en 2009 dans le canal du Loing à proximité de sa confluence avec la Seine (Fig. 3), constitue-t-elle un isolat ou une nouvelle voie d'accès du $C$. curvispinum?

II serait pertinent de suivre cette population et de réaliser des investigations plus en amont (canal du Loing) et en aval afin d'observer une éventuelle migration de $C$. curvispinum vers la Seine (Fig. 3).

- Quel est l'impact de C. curvispinum sur les populations d'invertébrés autochtones du bassin de la Seine?

Les données existantes sur les grands cours d'eau du bassin rhénan montre que l'introduction de $C$. curvispinum affecte principalement les populations de moule zébrée $(D$. polymorpha) (Paffen et al., 1994 ; Khalanski, 1997 ; Bachmann et al., 2001). Outre une compétition trophique (filtreurs), les tubes sablo-limoneux édifiés par $C$. curvispinum recouvrent les substrats durs qui deviennent impropres à la fixation des larves de $D$. polymorpha.

Dans le bassin de la Seine, sa présence ne semble pas s'accompagner 
de perturbation majeure des populations autochtones mais cet aspect mériterait d'être étudié plus en détail. II serait notamment intéressant d'étudier les populations d'Aselles (Asellus aquaticus), qui partagent le même habitat de prédilection (franges racinaires, blocs) que $C$. curvispinum. Une possible compétition entre ces deux espèces a déjà été envisagée dans le Rhône (Bij de Vaate et al., 2002).

\section{CONCLUSION}

Une espèce est qualifiée d'espèce exotique proliférante ou invasive lorsqu'elle parvient à étendre son aire de répartition depuis son point d'introduction et à atteindre de fortes densités (Devin, 2003).

La synthèse bibliographique des données hydrobiologiques existantes (141 stations) et la campagne de terrain menée en 2009 (29 stations) sur les voies navigables du bassin de la Seine ont mis en évidence une colonisation lente, modérée et disparate du bassin par $C$. curvispinum. Dix ans après les premières observations (1998), les populations installées se cantonnent aujourd'hui aux bassins de l'Oise, de l'Aisne aval et dans la Seine et la Marne aux environs de l'agglomération Parisienne.

II paraît donc exagéré en l'état, de qualifier $C$. curvispinum d'espèce invasive à l'échelle du bassin de la Seine. $\mathrm{Sa}$ vitesse de colonisation demeure nettement plus faible que celle observée dans d'autres bassins hydrographiques (Rhin, Meuse et Moselle) et, pour le bassin de la Seine, sans comparaison avec la fulgurante installation du Dikerogammarus villosus.

Ces premiers résultats mériteraient d'être approfondis (évolution des populations de $C$. curvispinum existantes, suivi des voies de colonisation, analyse des effets éventuels de la compétition inter-spécifique, ...). De façon plus générale, il serait également judicieux de suivre les populations de crustacés des grands cours d'eau du bassin de la Seine, qu'ils soient autochtones ( $G$. pulex, $A$. aquaticus) ou exogènes ( $G$. tigrinus, $D$. villosus, J. istrii ...) ; d'autres crustacés ponto-caspiens étant susceptibles d'envahir le bassin de la Seine à court et moyen terme (Beisel et al., 2006). Ces macro-invertébrés sont présents dans le réseau trophique et entrent dans le régime alimentaire de nombreuses espèces de poissons. Ainsi, le changement de communautés de macro-invertébrés et les variations d'effectifs sont des éléments qui peuvent directement impacter le réseau trophique et les populations piscicoles.

\section{REMERCIEMENTS}

Les auteurs remercient l'ensemble du personnel de la société Hydrosphère pour sa contribution au bon déroulement du projet et plus particulièrement $M$. Jérémy Leclere pour son implication au quotidien. Nos remerciements vont également à $M$. Jacques Mouthon (IRSTEA) et M. Mathieu Floury (EDF) pour leurs conseils avisés et la relecture de cet article. 


\section{RÉFÉRENCES}

Bachmann V., Usseglio-Polatera P., Cegielka E., Wagner, P., Poinsaint J.F. \& Moreteau J.C., 1997. Premières observations sur la coexistence de Dreissena polymorpha, Corophium curvispinum, et Corbicula spp. dans la rivière Moselle. Bull. Fr. Pêche Piscic. 344/345 : 373384.

Bachmann V., Beisel J.N., Usseglio-Polatera P. \& Moreteau J.C. 2001. Decline of Dreissena polymorpha in the River Moselle: biotic and abiotic key factors involved in dynamics of invasive species. - Archiv für Hydrobiologie 151 : 263-281.

Balyss D. \& Harris R., 1988. Chloride ion regulation in the freshwater amphipod Corophium curvispinum and acclamatory effects of external $\mathrm{Cl}^{-}$. J. Comp. Physiol. B 158 : 81-90.

Beisel J.N., Devin S., Moreteau J.C, Piscart C., Rousselle P. \& Usseglio-Polatera P., 2006. Macroinvertébrés invasifs et écosystèmes européens d'eau courante: dynamique, processus et outils de gestion. Rapport final du programme de recherches « invasions biologiques ».

Bij de Vaate A., Jazdzewski K., Ketelaars H.A.M., Gollasch S. \& Van der Velde G. 2002. Geographical patterns in range extension of Ponto-Caspian macroinvertebrates species in Europe. Canadian Journal of Fisheries and Aquatic Sciences $59: 1159-1174$.

Den Hartog C., Van Den Brink F.W.B. \& Van Der Velde G., 1992. Why was the invasion of the River Rhine by Corophium curvispinum and Corbicula species so successful? J. Nat. Hist. 26 : 1121-1129.

Dessaix J. \& Fruget J.F., 2008. Évolution des peuplements de crustacés du Rhône Moyen au cours des 20 dernières années, relation avec la variabilité hydroclimatique. Hydroécol. Appl. 16 : 1-27.
Devin S., 2003. Les invasions biologiques des milieux d'eau douce: Analyse du rôle des traits biologiques et écologiques d'un nouvel envahisseur (Dikerogammarus villosus, Gammaridae, Crustacea) dans le processus invasif. Thèse, Université de Metz, $193 \mathrm{p}$.

Genin B., 1992. Corophium curvispinum Sars, 1895 (Crustacea, Amphipoda) dans la Saône. Bull. Sci. Bourg. 45 : 27-32.

Herhaus K.F., 1978. Der erst Nachweis von Corophium curvispinum Sars, 1895 (Crustacea, Amphipoda, Corophidae) in Dortmund EMS-Kanal. Natur Heimat 38 (3) : 99-102.

Jazdzewski K., 1980. Range extension of some gammaridean species in Europe inland watercaused by human activity. Crustaceana Suppl. 6 : 84-107.

Jazdzewski K. \& Konopacka A. 2000. Immigration history and present distribution of alien crustaceans in Polish waters. In The biodiversity crisis and Crustacea, Proc. 4th Int. Crustacean Congress, Amsterdam, 20-24 July 1998, Vol. 2, edited by J.C. von Vaupel Klein and F.R. Schram, Brill, Leiden. Crustac. Issues, 12 : 55-64.

Kallenbach B., Montabaur D., Schleuter M., Gewässrekunde B. \& Coblence D., 2000. Le macrozoobenthos de la Moselle et de la Sarre - État des lieux Commissions internationales pour la Protection de la Moselle et de la Sarre (CIPMS).

Khalanski M., 1997. Conséquences industrielles et écologiques de l'introduction de nouvelles espèces dans les hydrosystèmes continentaux : la moule zébrée et autres espèces invasives. Bull. Fr. Pêche Piscic. 344/345 : 385-404.

Moon, H.P., 1970. Corophium curvispinum (Amphipoda) recorded again in the British Isles. Nature 226 : 976.

Paffen B.G.P, Van Den Brink F.W.B., Van Der Velde G. \& Bij De Vaate A., 1994. 
The population explosion of the amphipod Corophium curvispinum in the Dutch Lower Rhine. Water Sci. Technol. 29 (3) : 53-55.

Pygott J. \& Douglas S., 1989. Current distribution of Corophium curvispinum SARS Var. devium Wundsh (Crustacea, Amphipoda) in Britain with notes on its ecology in the Shropshire Union Canal. Naturalist 114 : 15-17.

Rajagopal S., Van Der Velde G., Paffen B.G.P. \& Bij De Vaate A., 1998. Ecology and impact of the exotic amphipod, Corophium curvipinum Sars, 1895 (Crutacea: Amphipoda), in the River Rhine and Meuse. RIZA, Lelystad, The Netherlands, $75 \mathrm{p}$.

Schöll F., 1990. Zur Bestandssituation von Corophium curvispinum Sars im Rheingebiet. Lauterbornia 5 : 67-70.

Van den Brink F.W.B., Van der Velde G. \& Bij de Vaate A., 1989. A note on the immigration of Corophium curvispinum Sars 1895 (Crustacea : Amphipoda), into the Netherlands via the River Rhine. Bul. Zool. Mus. Amsterdam 11 : 211-213.

Wouters K.A., 1985. Corophium curvispinum Sars, 1895 (Amphipoda) in the River Meuse, Belgium. Crustaceana 48(2) : 218-220. 